\title{
Automatic Passenger Detection in Safety Critical Mass Transit Environments
}

\author{
Ali Mroue, Marc Heddebaut, Fouzia Elbahhar, Atika Rivenq-Menhaj, \\ Jean-Michel Rouvaen
}

\section{To cite this version:}

Ali Mroue, Marc Heddebaut, Fouzia Elbahhar, Atika Rivenq-Menhaj, Jean-Michel Rouvaen. Automatic Passenger Detection in Safety Critical Mass Transit Environments. International Journal of Intelligent Transportation Systems Research, Springer Verlag, 2013, pp.10. hal-00928497

\section{HAL Id: hal-00928497 \\ https://hal.archives-ouvertes.fr/hal-00928497}

Submitted on 31 Jan 2014

HAL is a multi-disciplinary open access archive for the deposit and dissemination of scientific research documents, whether they are published or not. The documents may come from teaching and research institutions in France or abroad, or from public or private research centers.
L'archive ouverte pluridisciplinaire HAL, est destinée au dépôt et à la diffusion de documents scientifiques de niveau recherche, publiés ou non, émanant des établissements d'enseignement et de recherche français ou étrangers, des laboratoires publics ou privés. 


\title{
Automatic Passenger Detection in Safety Critical Mass Transit Environments
}

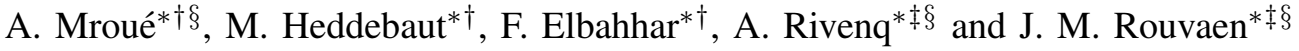 \\ * Univ. Lille Nord de France, F-59000 Lille, France \\ $\dagger$ IFSTTAR, LEOST, F-59666 Villeneuve d'Ascq, France \\ $\ddagger$ CNRS, UMR 8520, F-59650 Villeneuve d'Ascq, France \\ $\S$ UVHC, IEMN-DOAE, F-59313 Valenciennes, France
}

\begin{abstract}
Enhancing user safety constitutes a major issue in railway transport. In this paper, a novel solution for detection and identification of objects falling on railway tracks is proposed. This solution is based on a system using a set of consecutive ultra wideband (UWB) monostatic radars fed by a common transmission line. The main objective of this work is to study the different radiofrequency and signal processing subsets in order to evaluate and validate the full system. A slotted waveguide operating in its fundamental mode is used as the common transmission line. Slots are periodically perforated in the waveguide to constitute the radars. An optimal bandwidth and constant radiation coverage along the track are then optimized. The singularity Expansion Method (SEM) is used to characterize the objects falling on railway tracks. Complex Natural Resonances are then computed or measured and stored in a library. They are used in a specific discrimination process. Using both numerical simulations and experimental results, the discrimination process shows that human bodies are well detected and distinguished as well as other objects typically found on platform (suitcases, bottles...).
\end{abstract}

Index Terms-Fall on track, Passenger Detection/Identification, Discrimination, Radar Cross Section (RCS), UWB radar, Slotted waveguide, 3.9-5.4 GHz, Singularity Expansion Method (SEM), Complex Natural Resonance (CNR).

\section{INTRODUCTION}

Guided urban automated transportation systems are progressing significantly nowadays, highlighting many benefits. User's security and accessibility to these guided transport systems constitutes a major issue dealt with many teams [1]. In particular, this issue relates to interactions between passengers and the transport system [2]. Many dangerous situations can be envisaged in the guided transport environment. Among these situations, objects falling on track constitutes a significant problem. In the absence of sliding Platform Screen Doors (PSD), one main goal is to detect, in real time, objects falling from the platform to the track and to identify these objects. The detection and identification phases are then used to quickly decide to stop the trains in cases where an emergency situation is determined. The main goal is to enhance user safety and to maintain transport system availability, avoiding long traffic interruptions.

Most of the mass transit systems rely on operating rooms for command, control and supervision of the transport network. In these rooms, operators stand behind many screens to monitor user displacements. The effectiveness of these human-operator systems is limited, due to the large number of cameras to survey. Hence, it is clear that automatic devices must help operators to perform incident detection. Therefore, automatic incident detection helps to ensure a better security level, while reducing the rate of false alarm. This paper is focused on incidents related to track/platform interactions and, more particularly where objects fall on railway tracks [3].

Various devices are currently deployed to detect objects falling on track. Firstly, an approach consists in using PSD installed at subway stations [4]. They screen the platform from the track [5]. It is a very logical solution, since these doors prevent passenger access to the tracks; PSD open only when a train or subway is present. However, PSD are quite expensive and not easily implemented on some existing railway sites [6]. Despite their high level of performance, PSD lead sometimes to fatal accidents [7].

Other means to detect incidents can be grouped in two main categories: the passive method and the active method. The passive method uses passive sensors (usually one or several cameras attached to a machine vision hardware and software) to perform detection [8]. Cameras can be installed in the ceiling of the subway station. This solution has the advantage of providing a better resolution of the scene. However, image analysis requires significant computing resources. Moreover, this solution can suffer from occultation of the scene by disruptive elements, i.e. trains, but also from changing light conditions [9].

In active methods, detecting sensors send sound (sonar), radio (radar) or light (lidar) waves. Through the active method, distance of the target is deduced from the time-of-flight of the signal from the transmitter to the target and back. Some characteristics of the bounced signals are then used to identify the targets. Target position can also be determined using several distinct sensors to target time-of-flight.

In a detection system operating very close to the train equipment, sonar sensors can be interfered with by other potential ultrasonic signals generated, for example, by train compressor and braking systems. Lidar sensors can suffer from adverse environmental conditions, i.e. carbon dust generated by the brakes, rapidly covering the lidar lenses.

Some of these cited methods are expensive to implement (PSD) or exhibit some weaknesses. Therefore, R\&D efforts could be useful in this area. In this paper, a novel solution 
to detect and identify objects falling on railway track based on a set of radars is introduced [10]. Radars transmit a wave towards targets which return a signal called echo. The wave can be continuous or pulsed. It can be amplitude, frequency or phase modulated. In narrow-band radars, the received signal is usually similar to the transmitted one which in not the case of UWB pulsed radar. The signal is delayed and attenuated due to propagation channel. Noise combines with the received signal in the frequency range used.

Initially, radar was developed for military applications [11]. In terrestrial transportation, radars are mainly used to detect objects in front or all around vehicles. In railway transportation systems, they are used to improve railway safety and also to detect other trains ahead. The most popular radar families are correlation radar, Frequency Modulated Continuous Wave $(\mathrm{FM} / \mathrm{CW})$ radar and pulsed radar. As described in the following section, the pulsed radar technique is selected in this application to easily separate, in the time domain, radiations from different sources and signals coming from different closely spaced targets. Low implementation complexity is also generally associated to ultra wideband (UWB) pulsed radar systems.

According to the proposal, this paper is organized as follows: Section 2 introduces the designed radar system and deals with the process of optimization of the different system blocks. Section 3 describes tests carried out both in simulation and experimentation. Finally, some conclusions and perspectives are drawn in Section 4.

\section{DeteCtion DEVICE PRINCIPLE AND DESIGN}

The goal is to develop a system capable of being installed close to the track/rail interface, allowing an easy implementation along the track, and enabling identification of falling objects, especially passengers. For this purpose, a system using a set of ultra wideband radars fed by a common transmission line is used. This system combines pulsed radar techniques with a waveguide transmission line [12]. The waveguide is installed all along the platform. An UWB transmitter is connected at one extremity, a receiver and a signal processing unit are installed at the opposite side of the platform.

The system for detecting and identifying objects falling on railway tracks is described in Fig. 1. To explain its principle, let us consider a single slot perforated somewhere along the waveguide. Using the Babinet's principle, a slot perforated on a conductive plane can be considered as the dual-antenna of an electric dipole. This elementary antenna radiates part of the waveguide incident energy outside the waveguide and receives the bounced energy from the surrounding targets. Thus, the waveguide works as a low-loss transmission line propagating radiofrequency energy to and from this slot antenna. Therefore, we obtain a monostatic radar using this single slot antenna and a propagation line going to the transmitter plus a propagation line going to the receiver. The transmitted and the reflected signals are separated in the time domain. Now, as described in Fig. 1, let us consider several vertical slots perforated along the waveguide, for example every meter. As in the previous case of a single slot, each of these slots is equivalent to an elementary antenna. In the case of a continuous-wave (CW) transmitter, these slots would work as an end-fire array antenna. However, the system uses ultra wide band and short pulses. Thus, pulsewidth is shorter $(<1 \mathrm{~ns})$ than the equivalent travel time between two consecutive slots along the waveguide $(1 \mathrm{~m}$ or approximately $3 \mathrm{~ns}$ in the air). Then, pulse energy has left the preceding slot area when reaching the following one. Therefore, we obtain that energy is only feeding one slot at a time and we define a model using elementary consecutive radars. Consequently, each slot is considered as providing an elementary radar detection zone connected to the transmitter on one waveguide side and to the receiver on the opposite side.

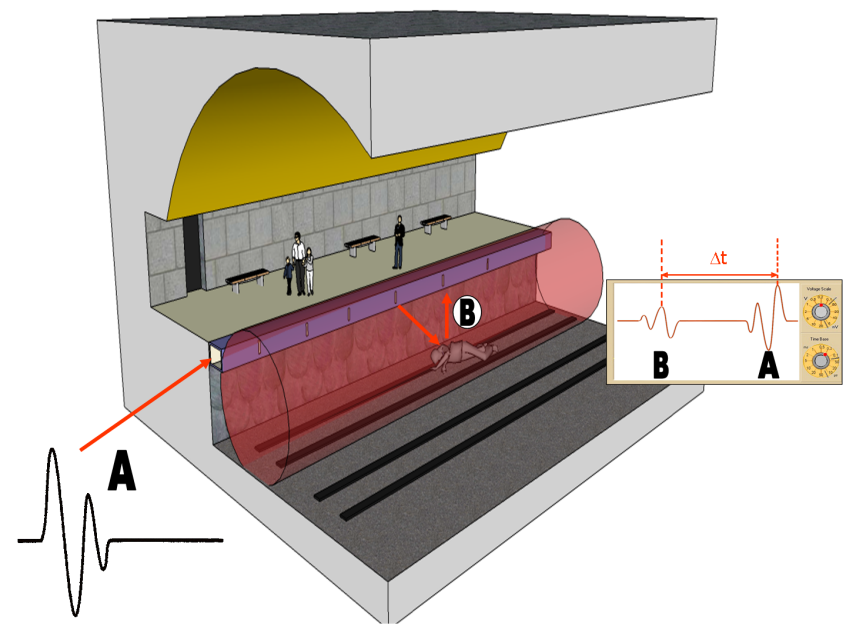

Fig. 1. Device for detection and identification of objects falling on railway tracks.

If we consider a rectangular aluminum waveguide with a cross section of $50 \times 25 \mathrm{~mm}$, the computed loss in its fundamental propagation mode is $0.037 \mathrm{~dB} / \mathrm{m}$ [13]. This figure compares favorably with copper coaxial cables. Therefore, a several tenths of meter- long waveguide can be installed along the platform, without experiencing excessive attenuation. As can be seen in Fig. 1, the waveguide is installed along the length of the platform edge, below the walking level. The slots are oriented to radiate towards potential objects falling on the track. This configuration optimizes the radar detection capability as well as limits the false alarms.

This system is composed of severals blocks: transmitter, propagation line and receiver. Firstly, the transmitter, which delivers pulses to the antenna, must be optimized in order to select the usable frequency band to emphasize the detection of selected objects. Secondly, the propagation line must also be optimized to allow good energy transfer along the track, with minimal attenuation and sufficient bandwidth. Discontinuities should be carefully selected to obtain a constant radiation coverage and a constant detection along the track, compensating progressively losses related to propagation line attenuation and the presence of upstream discontinuities. Finally, the receiver block aims to extract relevant information related to each type of obstacle in order to characterize and discriminate between them. Each of these blocks is discussed hereafter. 


\subsection{Optimization of usable bandwidth}

The objective is to increase passenger safety by promoting human detection and identification. Hence, a study on human Radar Cross Section (RCS) was carried out in order to determine the optimal bandwidth in which human bodies demonstrate high reflectivity (RCS) [14].

Three classes of human body are investigated (Fig. 2): an adult of $1.8 \mathrm{~m}$, a teenager of $1.2 \mathrm{~m}$ and a child of $0.6 \mathrm{~m}$. Two kinds of material are used: perfect electric conductor (PEC), as a purely simulation evaluation example, and muscle [15].

Simulations are carried out with an electromagnetic simulator. FEKO (FEldberechnung bei Koërpern mit beliebiger Oberfläche) is based on Method of Moments (MoM). In this case of application, FEKO uses hybridation of the MoM and Physical Optics method in order to reduce memory requirements and computation time.
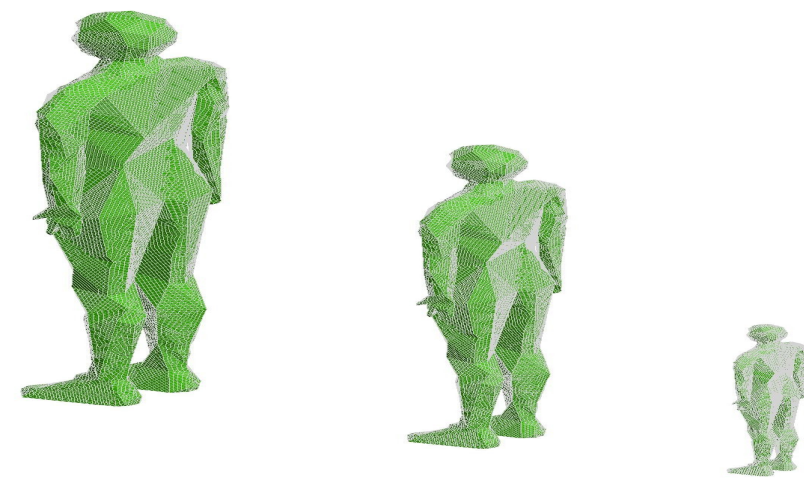

Fig. 2. Simulated human body: Adult, Teenager, Child.

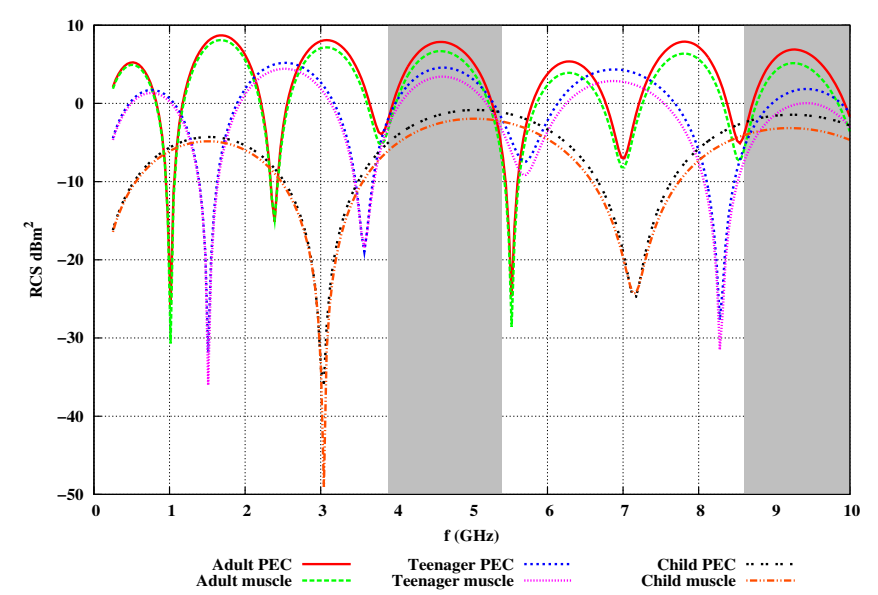

Fig. 3. RCS of human body: Adult, Teenager, Child.

As depicted in Fig. 3, RCS belonging to each class are grouped together. Gray areas in Fig. 3 correspond to frequency bands in which RCS is simultaneously high for the three classes. However, the last zone, i.e. [8.6 : 10] GHz, is not used to avoid high longitudinal losses in transmission line (discussed in the next section). Therefore, the optimal bandwidth is $[3.9: 5.4] \mathrm{GHz}$ with a central frequency $f_{0}=4.65 \mathrm{GHz}$. Afterwards, the transmission line optimization is based on this optimal frequency band. A further analysis has shown that this frequency band is also effective to optimize the target late-time response used for automatic target recognition (ATR).

\subsection{Study and optimization of radiated line}

As introduced in the previous section, the detection device for objects falling on tracks includes a progressive transmission line to convey signal energy from one end of the platform to the other, but also to create the successive monostatic radars equivalent to the presence of discontinuities. Optimization of the transmission line must allow simultaneously a reasonable longitudinal attenuation, compatible with the $100 \mathrm{~m}$ considered operational range, and significant radiation associated to the periodically inserted discontinuities, into a wide frequency range. Thus, some trade-off has to be defined. As obtained before, an optimized frequency band ranging from $3.9 \mathrm{GHz}$ to $5.4 \mathrm{GHz}$ is selected to promote human body identification. A comparative study was carried out in order to select the most appropriate line to use in our application. Waveguide longitudinal attenuation compares favorably with longitudinal attenuation provided by the microstrip line and the coplanar stripline (CPS). Furthermore, in the frequency band used, a rectangular metallic waveguide has cross-dimensions close to $5 \times 2.5 \mathrm{~cm}$. This low $2.5 \mathrm{~cm}$ thickness makes it convenient for an implementation all along the platform.

Once the waveguide technique is selected, the next task is to create the necessary discontinuities along the transmission line to allow radiation. This is obtained by perforating successive apertures or slots along a broad-wall of the waveguide.

2.2.1 Slotted Waveguide ( $S W)$ : To enable radiation, discontinuities in the form of "slots" are introduced along the waveguide broad-wall. The Finite-Difference Time-Domain (FDTD) was used by Ren et al. to present a full wave analysis of slots in the broad waveguide wall [13]. In this paper, a full wave approach bases on a numerical approach is also used to solve this particular problem. Numerical simulations are performed with the Computer Simulation Tool Microwave Studio 3-D electromagnetic simulator software (CST-MWS). It uses a Finite-Integral Time-Domain (FITD) method. To reach the requested frequency band, a rectangular waveguide is simulated. Its internal dimensions are $5 \mathrm{~cm}$ wide and $2.5 \mathrm{~cm}$ high. The conventional width to height ratio of 2 is chosen in order to maximize the usable bandwidth. To enable radiation outside the waveguide in free space, a slot is perforated in the broad-wall of the waveguide. Such an elementary slot is illustrated in Fig. 4.

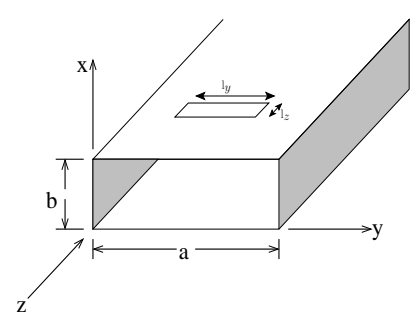

(a) (b)

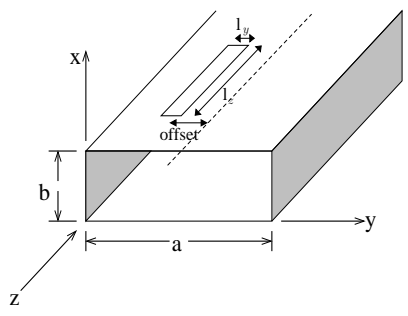

Fig. 4. Elementary slot perforation in waveguide broad-wall. 
The centered transversal slot (Fig.4(a)) is $l_{y}$ width and $l_{z}$ long with $l_{y} \gg l_{z}$. The slot has a perfectly rectangular shape, zero offset from the broad-wall centerline and transversal to the propagation direction. In the case of a longitudinal slot (Fig.4(b)), $l_{y} \ll l_{z}$, the slot is laterally displaced from the broad-wall centerline.

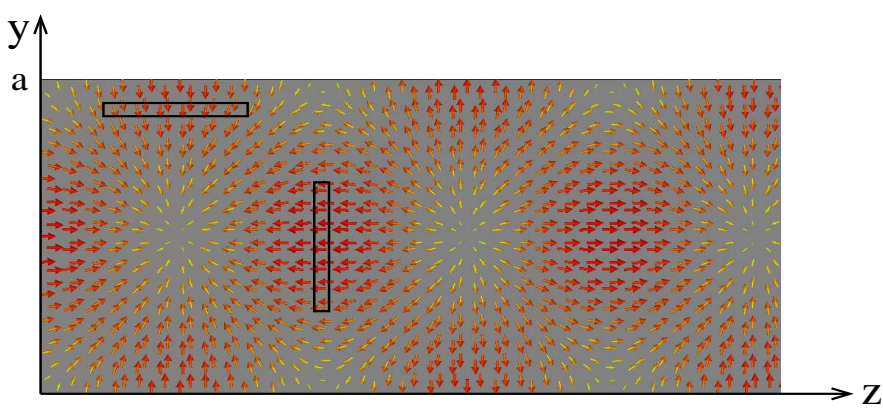

Fig. 5. Current distribution for the $\mathrm{TE}_{10}$ mode in the waveguide inner broad-wall and transversal and longitudinal slots.

In Fig. 5, the surface currents near the edges of the broadwall ( $y \approx 0$ or $y \approx a$ ) have only $y$ transverse components. Near the central line $(y \approx a / 2)$ the currents have only longitudinal $z$ components. Hence, longitudinal slots along the center line disturb no current lines. Consequently these slots do not radiate significant energy. Rather, centered transversal slots or longitudinal slots in the broad-wall near the wall edges will radiate.

In the next section, a design procedure aiming to optimize slot radiations for the two considered slot dispositions, i.e. transversal and longitudinal, is discussed. The goal is to create continuous radiation by each of the consecutive slots working as an elementary radar.

2.2.2 SW optimization procedure: If all transversal slots have the same length $l_{z}$ along the waveguide, then radiation is high near the input and very weak at the other end. This is due to energy lost in the transmission line and by radiation of the consecutive slots. Considering the above elements, to change the energy radiated by a longitudinal slot, the offset distance from the broad-wall centerline can be modified. To increase the energy radiated by a centered transversal slot, its great axis can be modified up to a resonant length in $\lambda / 2$.

To ensure continuous radiation coverage along the track, an optimization procedure is performed. This procedure is carried out according to the total efficiency parameter which characterizes the energy radiated by the slot. The total efficiency is the ratio of the radiated power noted $\left(P_{r}\right)$ to stimulated power noted $\left(P_{\text {stim }}\right)$ of each slot. Compared to the input power, the stimulated power considers any occurring reflections at the feeding location. The total efficiency $e$ is expressed as follows:

$$
e=\frac{P_{r}}{P_{\text {stim }}}
$$

The variation of the total efficiency according to the longitudinal slot offset (Fig. 6(a)) and to the transverse slot length (Fig. 6(b)) was numerically evaluated using CST-MWS simulator. As depicted in Fig. 6(b), a maximum efficiency close to $35 \%$ is obtained, slightly higher for the transverse slot.

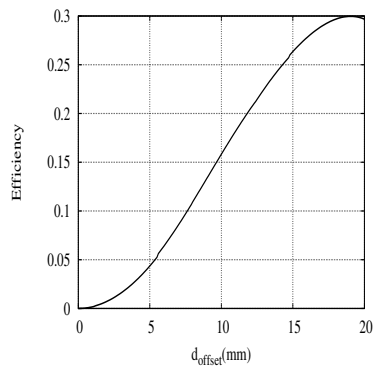

(a)

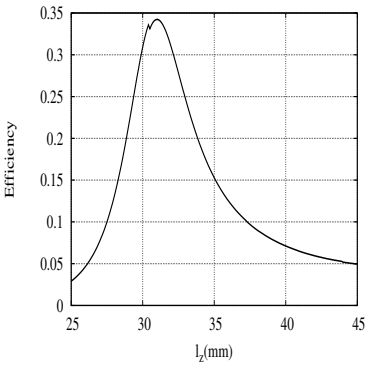

(b)
Fig. 6. Total efficiency variation according to the slot length and to the slot offset from the central line of the broad face.

Hence, using the relation between the available power and the radiated power that are governed by the desired efficiency, the following relations came about:

$$
\begin{array}{cc}
P_{r, 1}= & e_{1} P_{i n} e^{\left(-\alpha d_{i n}\right)} \\
P_{r, n}= & e_{n}\left[P_{i n} e^{-n \alpha d_{s l o t}}(1-\Gamma)^{(n-1)}\right. \\
& \left.-\sum_{k=1}^{n-1}(1-\Gamma)^{(n-k)} P_{k} e^{-(n-k) \alpha d_{s l o t}}\right] \\
& n=1, \ldots, N
\end{array}
$$

where $d_{i n}$ is the distance between the input waveguide port and the first slot, $P_{r, n}$ is the $n^{\text {th }}$ slot's radiated power, $e_{n}$ the total efficiency of the $n^{\text {th }}$ slot, $P_{i n}$ is the feeding power at the waveguide input port, $\alpha$ is the non-slotted waveguide longitudinal attenuation, $d_{\text {slot }}$ is the spacing distance between two consecutive slots, $\Gamma$ is the reflexion coefficient inside the waveguide, and $N$ is the total number of slots along the waveguide. In (3), the fraction in the square brackets is the stimulated power introduced in (1). By considering that $d_{i n}$ is equal to $\frac{d_{\text {slot }}}{2}$ and by setting

$$
P_{r, 1}=P_{r, 2}=\cdots=P_{r, n}=P_{r, n+1},
$$

a backward recursive expression according to the total efficiency is obtained:

$$
e_{n}=\frac{e_{n+1} e^{-\alpha d_{s l o t}}(1-\Gamma)}{1+e_{n+1} e^{-\alpha d_{s l o t}}(1-\Gamma)}
$$

An assumption is used to initialize the recursive equation in (5) i.e. most of the remaining power must be radiated by the last slot. In other words, $e_{N}$ is set to the maximum in Fig. 6(a) and the length $l_{z}$ of each slot is set so that $e_{N}$ is maximum in Fig. 6(b). Fig. 6(a) and (5) are conjointly used to determine the offset noted $d_{\text {offset }}$ of each slot. In practice, to ensure a constant human body detection along the structure, slots are spaced by a distance $d_{\text {slot }}=2 \mathrm{~m}$. Considering short pulses of 1-2 ns long, radiofrequency energy has left the preceding slot before reaching the next one. Hence, a deduction can be made that the slotted waveguide is working as a succession of independent radiation sources.

2.2.3 Design example of a vertically polarized $S W$ : Let us consider a $5 \mathrm{~m}$ long waveguide example. The design parameters have been set to $d_{\text {slot }}=2 \mathrm{~m}$, the attenuation constant to $3.7 \mathrm{~dB} / 100 \mathrm{~m}$. The total slot efficiencies are given in Table I. 
TABLE I

DISTRIBUTION OF THE TOTAL SLOT EFFICIENCY.

\begin{tabular}{lllll}
\hline \hline$n$ & 1 & 2 & 3 & 4 \\
\hline$e_{n}$ & 0.13 & 0.17 & 0.22 & 0.35 \\
\hline
\end{tabular}

Once computed, Table I and Fig. 6(a) are used to calculate lateral offsets $d_{\text {offset }}$ of each slot. The obtained slot offsets increase from slot to slot (6) compensating for the loss of power due to the preceding slot radiations.

$$
d_{\text {offset }, 1}<d_{\text {offset }, 2}<d_{\text {offset }, 3}<d_{\text {off set }, 4}
$$

By using the optimization procedure, introduced in the previous section, the radiated power from this slotted waveguide is depicted in Fig. 7.

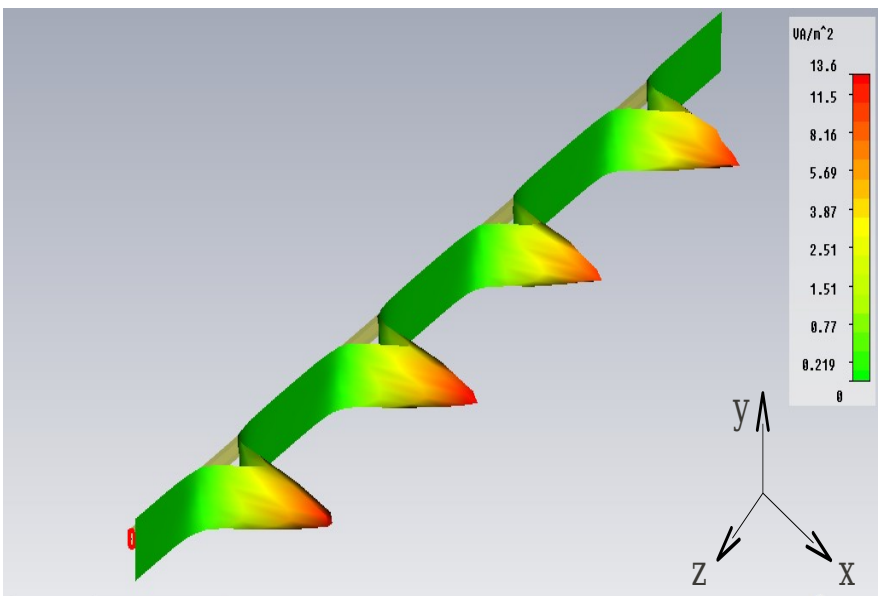

Fig. 7. Radiated power from the optimized longitudinal slots.

In the time domain, radio-frequency energy appears successively along the consecutive slots of the waveguide. Recording the slot maximum radiation along the waveguide shows an almost constant maximum radiated signal.

The far-field pattern of the vertically polarized slot is presented in Fig. 8.

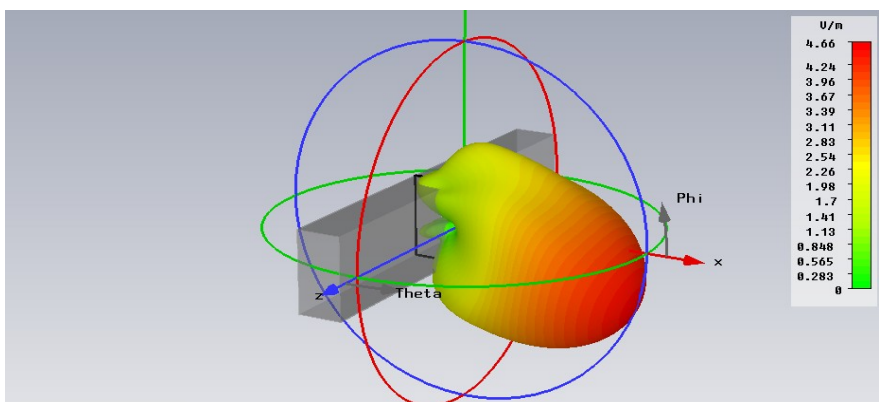

Fig. 8. Far-field pattern of one centered longitudinal slot.

In this first part of the paper, an operating frequency band and a corresponding antenna structure were selected and optimized. In the second part, the target recognition procedure is presented.
2.2.4 Radar power requirement: There is limited available frequency and demands for communications, and radar-based sensors may continue to grow. Any electronic system which permits the same frequency band to be shared in the same location without interference could probably be used over medium or long term period of time if the interference can be kept as low as possible, regardless of present or future spectral allocations and emission restrictions in various regions of the world. Considering this particular radar application, the total input power introduced into the slotted waveguide is evenly distributed into the different slots, along the length of the platform. Consequently, the locally radiated power per each slot is maintained to a low value. Moreover, the detection range of the radar is limited, typically to a few meters or the width of the track. This necessitates a limited power, comparable to the one used in unlicensed radars which detects the presence of objects entering a protected area. Finally, a low pulse repetition rate, between 1 and $10 \mathrm{~ms}$, is sufficient to detect all the limited motion movements in front of the radar. Low-data-rate transmitters are typically peak power limited and not mean power limited by the different regional telecommunication regulators. Some regulators allow UWB to use a higher power for UWB systems using low repetition rates. In the United States, the peak power may not exceed $0 \mathrm{dBm}$ at the UWB signals center frequency in a $50 \mathrm{MHz}$ resolution bandwidth. During the experiments presented in section 3.2, the power used by the Vector Network Analyzer, was set to $5 \mathrm{dBm}$ (3 mW) while sweeping a $1 \mathrm{GHz}$ into 128 acquisitions of the analyzed band.

\subsection{Automatic Target Recognition (ATR) System}

The proposed UWB radar aims to recognize automatically a target in its vicinity. The identification of the target nature will be realized by the comparison between the deduced target properties and the different target characteristics already recorded in a database. These properties are called target singularities. In other words, these singularities are the spatial repartition of called "bright spots" on the target surface. Two possible processing methods for target identification and recognition are discussed.

The first method is based on radar reconstruction of images to represent the detected targets. In this work environment, automatically discriminate between targets is seeked, while reducing the processing volume and emphasizing a good discrimination. Therefore, an Automatic Target Recognition (ATR) processing method is preferred. This method exploits all the information contained in signal echoes. The ATR method consists of three successive stages: extraction, feature selection, and discrimination.

The main drawback of the ATR method is that the singularities vary as the incident aspect changes. Target singularities are not a priori known. Therefore, it is preferable to implement a technique that extracts target characteristics that are purely dependent on the target itself and independent of the incident aspect angles.

2.3.1 Singularity Expansion Method: The general principle of the chosen ATR method is the following: it has been 
demonstrated that the late time portion of an impulse response from a radar target can be expressed as a summation of a finite number of damped oscillations with independent coupling. Each damped oscillation is representative of an intrinsic characteristic of the target and is associated with a mathematically equivalent Complex Natural Resonance (CNR) [16]. A library of CNR sets, corresponding to commonly found objects falling on tracks, is built. Then, for any new fall on track received signal, we extract a corresponding new set of CNR. If a sufficient number of extracted CNR, corresponding to an existing set of CNR in the library is then found, the formal identification of the target is obtained.

To explain this principle in more detail, let us remember that when a broadband signal, corresponding to a far-field illuminating signal, hits a target, it gives rise to induced currents on its surface. Charges circulate along this surface to establish electrical neutrality. Then, by dispelling energy in the form of radiation or heat, a state of equilibrium is restored. Resonances occur if the broadband signal includes the resonance domain of the target. The resonance domain corresponds to electromagnetic wavelengths of the same order as the objects dimensions. These resonances are only dependant on intrinsic characteristics of the target, i.e. dielectric properties and physical geometries; they do not depend of the angle of incidence of the illuminating signal. Therefore, this method overcomes any angle dependency limitation. As a consequence, a passenger falling on the track in front of such a radar will be detected as a human being, whatever his posture. This method is called resonance-based radar target recognition. As the name states, it characterizes the radar target based on the Complex Natural Resonance introduced by Baum [16]. As explained before, CNR are purely dependent on the physical properties of the target and independent of the incident angle [16] and incident wave polarization [16], [17]. The development of resonance based radar target recognition uses the Singularity Expansion Method (SEM) also introduced by Baum [3]. Baum was inspired by observing that the typical transient response of various objects behaves as a sum of exponentially damped sinusoids. Using (7), Baum proposes a model in which the transient response of a target is composed of two successive parts. The impulsive part, corresponding to direct reflection of the incident wave on the target surface, will be received first. The impulsive part is followed by an oscillating part, which corresponds to late time response and is due to resonances phenomena of the target characteristics. The CNR are embedded in the oscillating part of the signal, which is modeled as follows:

$$
y(t)=h(t)+b(t) \approx \sum_{n=1}^{M} R_{n} e^{s_{n} t}+b(t)
$$

where,

- $s_{n}$ is the $n^{t h}$ natural resonance coefficient (pole) of the target: $s_{n}=\sigma_{n}+j \omega_{n}$.

- $\sigma_{n}$ is the damped factor due to losses on the surface and inside the target.

- $\omega_{n}=j 2 \pi f_{n}$ with $f_{n}$ natural frequency.

- $R_{n}$ the $n^{t h}$ residue

\section{- $b(t)$ is an additive noise}

2.3.2 CNR Extraction Techniques: A few years after the proposal of the SEM, Van Blaricum and Mittra [18] proposed the use of the Prony method for extracting the CNRs and residues from the late time response. The main drawback with the Prony method is that it is highly sensitive to noise. The Generalized Pencil of Function method (GPOF) was then introduced. Combined with the Singular Value Decomposition (SVD) technique, it becomes the Total Least Square Matrix of Pencil Method (TLS-MPM). Compared to Prony method, TLS-MPM is not only more computationally efficient, but it also has better statistical properties for CNR estimation. SVD is used to give a prediction of the number of CNR and also to remove noise from the sample data.

\section{Results}

In previous sections, the different components of the system were defined and optimized. SEM treatment of various targets usually found in railway platform environments is now presented. For each type of target, CNRs are extracted for the further discrimination procedure. First, a simulation validation is carried out and, then, an experimental validation is performed.

\subsection{Simulation results}

The detection scene is simulated using CST-MWS. The detection device consists of a slotted waveguide excited at one end by a waveguide port. It is working in the $T E_{10}$ fundamental mode. At the other end, another waveguide port is located to collect the signals directly propagated from the generator and returned from the target. To ensure, that a plane wave hits the target, the target is placed at a distance $12 \lambda$ in front of the slot, where $\lambda$ is the central used wavelength. The generated wave propagates inside the waveguide up to the slot. Once it arrives at the slot level, a part of its energy is radiated outside the waveguide, a second part is reflected to the generator and a last fraction continues propagating up to the receiving port. The radiated part propagates in free space up to the target. The target diffracts the incident wave with a delayed part (late time signal) corresponding to the resonance phenomena following the diffracted components. The reflected components coming from the target are coupled inside the waveguide through the slot and propagate up to the receiving port. For the SEM treatment, received components that correspond to the resonance phenomena are considered. Before extraction of the CNRs, collected data must be prepared. Data preparation is in three steps. The first step consists in deconvolving the received signal $h(t)$ from a static response $h_{\text {stat }}(t)$ corresponding to data received without a target. This deconvolution aims to eliminate CNRs specific to the excitation wave and to the antenna (waveguide), in order to then emphasize the target response.

Once singularities outside the target are eliminated, the second step consists in filtering the target response in order to remove residual high frequency components corresponding to noise. Noise origin may be mathematical in the case of simulation or thermal in the case of experimentation. 
The last step of preparation consists in determining the beginning of the late time $T_{L}(8)$ that is crucial in the CNR extraction process.

$$
T_{L}=\frac{2 L_{c}}{c}+2 * T_{v}+T_{p}
$$

where, $L_{c}$ is the largest dimension of the target, $T_{v}$ is the time-of-flight between the antenna and the target and $T_{p}$ is the excitation pulse width.

The human body is the main priority of the detection system. Therefore, it is compared to other targets in order to distinguish it. Other targets, i.e. objects that might fall from platforms onto railway tracks, belong to passengers. In this study, different obstacles are considered: travel luggage, suitcases, briefcases, bottles etc.

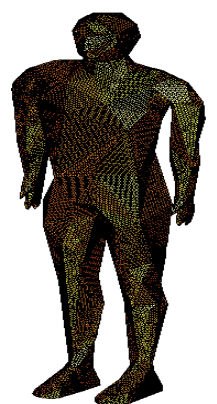

(a) Human body.

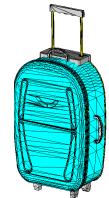

(b) Travel luggage.

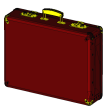

(c) Briefcase.

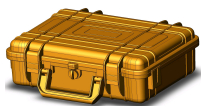

(d) Metal case

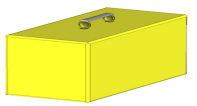

(e) Metal box.

Fig. 9. Obstacles existing in railway environment.

The human body numerical model is designed using a 3D designer and then imported into CST-MWS as a space mesh. Several models of human body were analyzed: an adult $(1.8 \mathrm{~m})$, a teenager $(1.2 \mathrm{~m})$ and a child $(0.8 \mathrm{~m})$. Human models have homogeneous bodies of PEC or muscles. PEC is used only for the simulation purpose. In this paper, teenager PEC results are highlighted. The teenager model is placed in front of the slot at a distance of about $12 \lambda$ (Fig. 10). After the above described preparation stages, the teenager response is illustrated in Fig. 11. The zoom illustrates the late time response corresponding to the resonance phenomena.

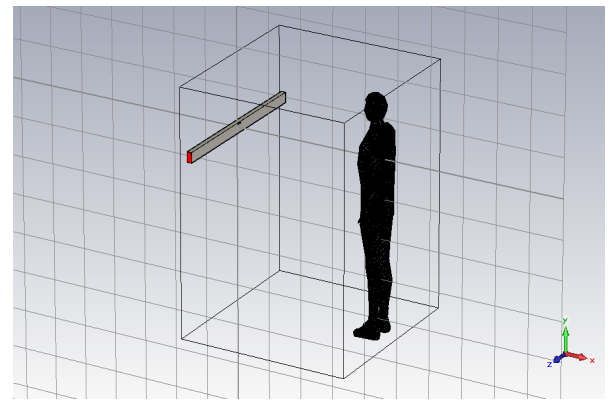

Fig. 10. Teenager numerical model incorporated in the simulation scene.

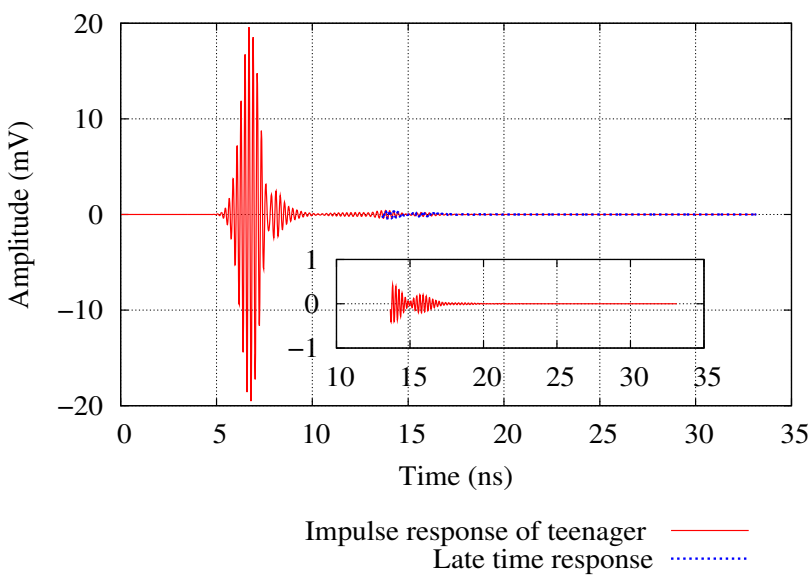

Fig. 11. Impulse response of teenager model with a zoom on the late time signal.

Once the CNR are extracted (Fig. 12) using TLS-MPM, they are processed by the discrimination procedure consisting in comparing the mapping of teenager PEC with all mappings already stored in a database. An intuitive idea is to associate the CNR in the mapping test to their neighbors in the other (counterparts). Then, the euclidean distance is calculated between the CNR and their counterparts. Hence, each comparison results in a set of vectors of distance, i.e. each vector contains distances between the CNRs of the mapping test and their counterpart in another stored mapping. These distances are normalized by the maximal distance between the CNRs and their counterparts in other mappings.

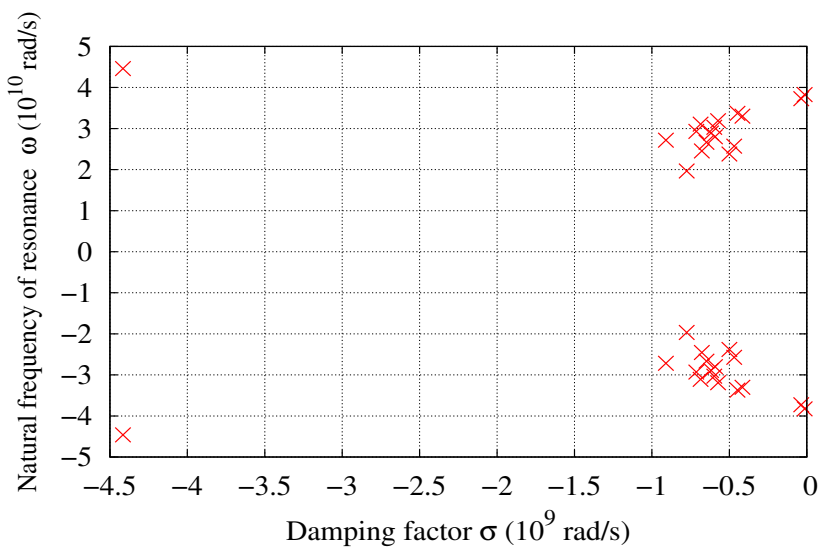

Fig. 12. CNR mapping in complex plane belonging to teenager model.

Discrimination results between obstacles stored in the database are depicted in Table II. The detected objets are placed in the first column of Table II and the stored objects in the database are placed in the first row.

Each line in Table II contains a grayed cell. This cell indicates that obstacles situated in the first cell of the corresponding column is the most similar to the obstacle contained in the first cell of this line, i.e. "teenager" is an obstacle similar to "adult" and vice versa. The zero value is trivial, since each obstacle is similar to itself. The "teenager muscle" 
International Journal of Intelligent Transportation Systems Research, May 2013, Volume 11, Issue 2, pp 87-100, @ Springer, DOI:10.1007/s13177-013-0059-7 http://link.springer.com/journal/13177

TABLE II

ILLUSTRATION OF DISCRIMINANT PARAMETERS FOR EVERY STORED OBSTACLES WITH INDICATION OF THE MOST SIMILAR OBJECT (GRAYED CELL).

\begin{tabular}{|c|c|c|c|c|c|c|c|c|c|}
\hline & Teenager & Teenager muscle & Adult & Child & Child muscle & Metal case & Briefcase & Metal box & Travel luggage \\
\hline Teenager & 0 & 0.059 & 0.039 & 0.055 & 0.077 & 0.099 & 0.084 & 0.1 & 0.071 \\
\hline Teenager muscle & 0.091 & 0 & 0.12 & 0.1 & 0.049 & 0.14 & 0.12 & 0.17 & 0.1 \\
\hline Adult & 0.021 & 0.042 & 0 & 0.031 & 0.031 & 0.026 & 0.037 & 0.034 & 0.043 \\
\hline Child & 0.022 & 0.037 & 0.033 & 0 & 0.023 & 0.026 & 0.034 & 0.032 & 0.032 \\
\hline Child muscle & 0.01 & 0.0045 & 0.0061 & 0.0042 & 0 & 0.0073 & 0.006 & 0.0087 & 0.008 \\
\hline Metal case & 0.36 & 0.36 & 0.17 & 0.16 & 0.25 & 0 & 0.19 & 0.093 & 0.18 \\
\hline Briefcase & 0.0096 & 0.011 & 0.0089 & 0.0074 & 0.0073 & 0.0067 & 0 & 0.0077 & 0.0072 \\
\hline Metal box & 0.0098 & 0.012 & 0.0079 & 0.007 & 0.011 & 0.0033 & 0.0077 & 0 & 0.0054 \\
\hline Travel luggage & 0.0083 & 0.007 & 0.0097 & 0.0067 & 0.0094 & 0.006 & 0.0069 & 0.0052 & 0 \\
\hline
\end{tabular}

similar to the "child muscle" indicates that the discrimination system is able to identify objects composed of the same material. "Child" PEC is similar to "adult" (0.022) and to "child muscle" (0.023). The bags also constitute an interesting category. They are grouped together.

As conclusion of this simulation stage, this particular radar offers good results since it can discriminate a human body from a set of obstacles grouping several human bodies and several kind of bags.

\subsection{Experimental results}

In a real railway platform environment, the local electromagnetic noise can be significant, potentially disturbing electronic equipment. However noise power spectrum density is generally high at low frequency but significantly reduced above $1 \mathrm{GHz}$. Consequently, the use of radar operating at several $\mathrm{GHz}$ helps obtaining a sufficient signal to noise ratio. Furthermore the waveguide is a well-shielded transmission line cutting-off the signals below $3 \mathrm{GHz}$ and the slots are optimized to radiate signals at several $\mathrm{GHz}$. At low frequencies, they operate as very selective high-pass filters reducing the level of coupling between the railway platform environment noise and the inside environment of the waveguide. Non-useful reflections coming from fixed reflectors present in the platform scene, such as walls, track equipment..., cannot be avoided. These reflections appear as constant signals in the received radar signal. There are two ways to manage these unwanted signals. The first consists in limiting the time window of observation to realistic values. For example, it is expected that a passenger can fall close to the platform edge, but can certainly not jump several meters across. Therefore, the useful processed time window can be limited to a short value or to an equivalent limited propagation range. For example, at the speed of light, a 10 ns time window would correspond to an overall distance of $3 \mathrm{~m}$ or to obstacles up to $1.5 \mathrm{~m}$ away. Any obstacle farther than this distance will not be considered. The second solution consists in calibrating the system in absence of any fall on track situations. This received radar signal is recorded as a calibration file. It contains all the nonnecessary reflected signals. Subsequent received signals are then subtracted from this calibration signal. Therefore, all the non-useful fixed reflections disappear, leaving only the relevant data.

In order to validate the designed system experimentally, tests were carried out in an anechoic chamber environment. The dimensions of this chamber are: $7 \times 7 \times 3 \mathrm{~m}$. For this experimentation the waveguide is $3 \mathrm{~m}$ long. At one end, the waveguide is connected to a Vector Network Analyzer (VNA) port (HP 8720D $50 \mathrm{MHz}-20 \mathrm{GHz}$ ). The other end is connected to the other VNA port (Fig. 13). The connections between the waveguide ends and the VNA are performed using coaxial/waveguide transitions (Fig. 14). Using the

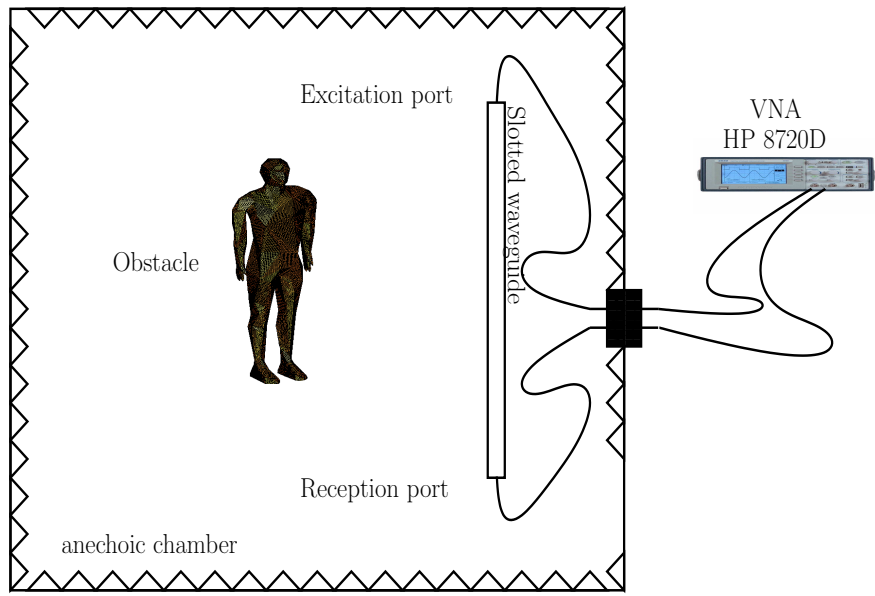

Fig. 13. Geometrical configuration of detecting device inside the anechoic chamber.

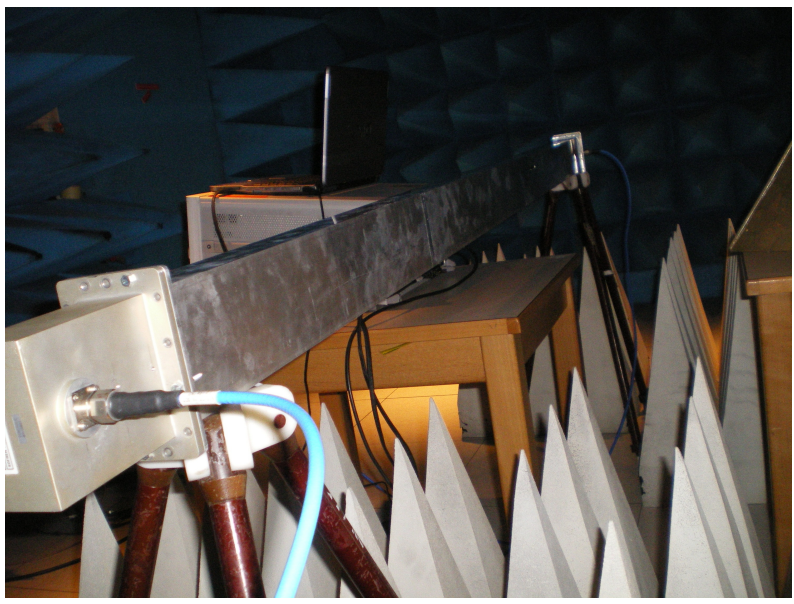

Fig. 14. Measurement configuration in the anechoic chamber.

VNA, frequency-domain measurements are performed which provide some flexibility during the processing steps. In the case of time-domain measurements, the tested frequency band is defined by the excitation waveform and cannot be easily 
modified during the processing steps. Initially, using the VNA, a wide analysis frequency analysis band can be selected and then progressively reduced by considering fewer measurement points. In practice, a $1 \mathrm{GHz}$ band is chosen and 1600 points are acquired for each measurement in this band. The transmission power is set at $5 \mathrm{dBm}$. One longitudinal slot and one transversal slot have been perforated in the waveguide broadwall. Although experiments were conducted with two types of slots: transversal and longitudinal slots, in this paper, only results obtained with the longitudinal slot are presented. In these tests, different obstacles are used. Three human bodies: a woman of $1.65 \mathrm{~m}$ and two men (man 1 and man 2) of $1.7 \mathrm{~m}$ and $1.8 \mathrm{~m}$ respectively. Two bags of different contents and sizes are also analyzed. Bag 1 is made of plastic with dimensions of $0.48 \times 0.3 \times 0.1 \mathrm{~m}$. Bag 2 is made of plastic also and has a metal frame. Man 1 is measured from the front and from the back. For the bag measurements, different angles of incidence are used: $0^{\circ}, 45^{\circ}$ and $90^{\circ}$. Obstacles are placed at $0.3 \mathrm{~m}$ in front of the slot. In Fig. 15 results corresponding to man 1, measured from the front, are presented. The frequency response of man 1 is smoothed in order to reduce the additive noise effect (Fig. 16), which induced a smoothed impulse response (Fig. 17). The major information is contained in a reduced frequency band. Other frequencies contain erroneous CNR and have been eliminated by a filtering procedure. CNR mapping of man 1 is depicted in Fig. 18.
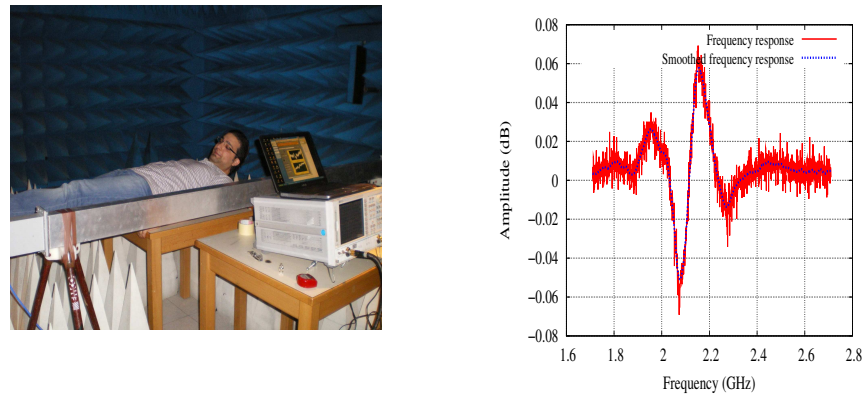

Fig. 15. Image illustrating man 1's measurement in anechoic chamber.

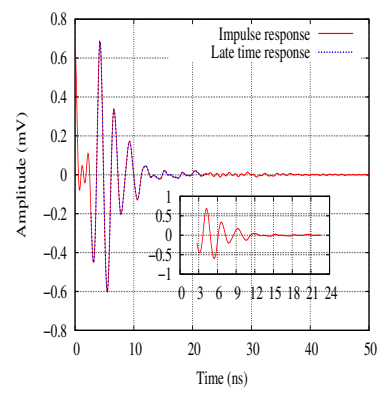

Fig. 17. Impulse response and late time response.

Once CNRs are determined from obstacles cited above, the discrimination procedure begins. By this procedure, a maximum of similarity between these obstacles is measured.
Table III presents discrimination parameters obtained using different obstacles.

First of all, human bodies dealt with in these experiments were isolated from other selected obstacles. In other words, the presented discrimination method is able to clearly identify and to successfully distinguish a human body among a set of different obstacles, which is the aim of this detecting and identifying system. Furthermore, all bags are grouped together. Bag 1 is recognized whatever the angle of incidence. The same conclusion applies to bag 2. Post-processing was performed in Matlab ${ }^{\mathrm{TM}}$ and time processing is about 5 seconds. In the future, this device could be implemented on FPGA cards to significantly improve the processing time in order to be effectively used on a platform.

\subsection{Performance estimation}

When estimating the performance of a radar detection system, both the probability of detection and the probability of false alarm are important parameters. At this stage of the study, such a detailed analysis was not performed but will be considered for future work. However, this paragraph discusses the parameters which were initially considered to be of prominent importance in obtaining a good detection probability and a low probability of false alarm.

Many different objects can be found falling on railway tracks. Our efforts have been put into detecting human beings; therefore the frequency band has been optimized to achieve an optimized detection. Other objects of lower radar cross sections (RCS), little pets for example, may not be detected as efficiently. Moreover, as explained in 2.3, the use of the ATR method overcomes the angle dependency limitation. As a consequence, a passenger falling on the track in front of the radar will be detected as a human being, whatever his posture. This enhances the capacity of the passenger detection system. Let us now consider the identification problem. We have indicated in 2.3.1 that a library of sets of CNR, corresponding to commonly found objects falling on tracks was built. For any new fall on track-received signal, corresponding new CNRs are extracted. They are compared to the existing library sets of CNR stored. If a sufficient match is then found, the formal identification of the target is obtained. It is then possible to use a low computation time by using a small number of CNR. Using a small number of considered CNR, the identification will be fast but false identifications may occur and consequently false alarms can be generated. If we use a higher number of CNR, the identification will be better but obtaining a real time functioning will be more difficult. Therefore, a satisfactory trade-off has to be carefully selected.

\section{Conclusion}

Interactions between passengers and the transport system are numerous; some of them are particularly critical. Many dangerous situations can be envisaged in the guided transport environment. Among these situations, objects falling on track constitute a significant problem. A novel solution for the detection and discrimination of objects falling on railway tracks has been proposed. This solution is based on a set of consecutive 
International Journal of Intelligent Transportation Systems Research, May 2013, Volume 11, Issue 2, pp 87-100, @ Springer, DOI:10.1007/s13177-013-0059-7 http://link.springer.com/journal/13177

TABLE III

ILLUSTRATION OF DISCRIMINANT PARAMETERS FOR EVERY STORED OBSTACLES WITH INDICATION OF THE MOST SIMILAR OBJECT (COLORED CELL).

\begin{tabular}{llllllllllll}
\hline & $\begin{array}{l}\text { Man } \\
\text { front }\end{array}$ & $\begin{array}{l}\text { Man } \\
\text { back }\end{array}$ & Woman & Man 2 & $\begin{array}{l}\text { Valise } \\
45^{\circ}\end{array}$ & $\begin{array}{l}\text { Bag } \\
1-90^{\circ}\end{array}$ & $\begin{array}{l}\text { Bag } \\
1-0^{\circ}\end{array}$ & $\begin{array}{l}\text { Bag } \\
2-45^{\circ}\end{array}$ & $\begin{array}{l}\text { Bag } \\
2-0^{\circ}\end{array}$ & $\begin{array}{l}\text { Bag } \\
2-90^{\circ}\end{array}$ \\
\hline Man 1-front & 0 & 0.037 & 0.045 & 0.07 & 0.044 & 0.079 & 0.044 & 0.066 & 0.091 & 0.065 \\
Man 1-back & 0.035 & 0 & 0.038 & 0.079 & 0.056 & 0.061 & 0.073 & 0.1 & 0.099 & 0.13 \\
Woman & 0.045 & 0.034 & 0 & 0.051 & 0.06 & 0.074 & 0.089 & 0.092 & 0.098 & 0.063 \\
Man 2 & 0.04 & 0.036 & 0.023 & 0 & 0.052 & 0.05 & 0.047 & 0.046 & 0.06 & 0.044 \\
Bag 1-45 & 0.1164 & 0.1528 & 0.1235 & 0.1536 & 0 & 0.0698 & 0.0471 & 0.1322 & 0.2318 & 0.2444 \\
Bag 1-90 & 0.1506 & 0.1422 & 0.1923 & 0.1265 & 0.0683 & 0 & 0.1146 & 0.1651 & 0.998 & 0.2272 \\
Bag 1-0 & 0.1886 & 0.1602 & 0.2045 & 0.2259 & 0.0683 & 0.1699 & 0 & 0.16 & 0.2523 & 0.2827 \\
Bag 2-45 & 0.1375 & 0.09 & 0.2313 & 0.2294 & 0.2522 & 0.2468 & 0.1048 & 0 & 0.1523 & 0.0868 \\
Bag 2-90 & 01554 & 0.1918 & 0.266 & 0.249 & 0.334 & 0.325 & 0.117 & 0.1406 & 0.069 & 0 \\
Bag 2-0 & 0.1258 & 0.898 & 0.2015 & 0.1667 & 0.2565 & 0.346 & 0.1104 & 0.2122 & 0 & 0.0593 \\
\hline
\end{tabular}

electromagnetic detection and identification areas fed by a common high frequency transmission line. In this paper, the different subsets of the equipment have been studied and optimized. An optimal bandwidth and constant radiation coverage have been identified. Several types of obstacle commonly found on platforms have been analyzed; considering this specific application, human body detection and identification have been emphasized. The Singularity Expansion Method was used to characterize these obstacles and the Total Least SquareMatrix of Pencil Method was applied to extract the Complex Natural Resonances of the obstacles. In order to discriminate between these obstacles, a specific discrimination method was developed. This system was numerically simulated and also experimentally evaluated. Both simulation and experimental results show that the detection and discrimination processes are effective. A human body was always found to be similar to other human bodies recorded in the library. Bags were all identified to belong to the same distinct bag-class. Furthermore, the detection/identification procedure showed providing a high capacity to identify obstacles built with the same material (case where teenager muscle was found similar to child muscle). A prototype was developed to show the feasibility of the designed system and experimental results demonstrated good reliability. On dual track platforms, a system is used per track. Since the transmitted pulses are very short, the analyzed time window is short and the pulse repetition frequency is low, the two track distinct systems are simply desynchronized to avoid interference between them. Future work is directed at reducing the processing time by transferring the developed off-line simulation tool on an integrated circuit designed to be configured by the engineer, after manufacturing. Although initially designed to be implemented on platforms, other applications of the system, enhancing the detection of human intrusion in tunnels or along dark corridors are also currently considered.

\section{REFERENCES}

[1] J. E. Anderson, "Safe design of personal rapid-transit systems," Safety Science, vol. 19, no. 2-3, 1995.

[2] W. H. K. Lam, C. Y. Cheung, and Y. F. Poon, "A study of passenger discomfort measures at the hong kong mass transit railway system," Journal of Advanced Transportation, vol. 33, no. 3, pp. 389-399, 1999. [Online]. Available: http://dx.doi.org/10.1002/atr.5670330308

[3] R. Kasai, "Safety measures against fall accidents on platforms," Japanese railway engineering, vol. 43, no. 1, 2003.

[4] B. Cooke, "Sliding Doors on Platform Edge," The Railway Magazine (Westminster: Tothill Press), 1959.
[5] M. Miyachi, Y. Sasaki, K. Morisaki, K. Onda, and M. Miyaoka, Safety and Security in Railway Engineering. WIT Press, 2010, ch. Security at the platform, pp. 9-18.

[6] C. Law, P. S. Yip, W. S. Chan, K.-W. Fu, P. W. Wong, and Y. Law, "Evaluating the effectiveness of barrier installation for preventing railway suicides in hong kong," Journal of Affective Disorders, vol. 114, no. 13, pp. 254 - 262, 2009. [Online]. Available: http://www.sciencedirect.com/science/article/pii/S0165032708003212

[7] I. H. Tribune, "Man caught between subway train and safety doors dies in Shanghai," International Herald Tribune, 032009.

[8] K. IKO, "The safety: Application of image-processing technologies to platform gate sensors," Railway \& Electrical Engineering, vol. 16 no. 10 , pp. 17-23, 2005.

[9] L. Khoudour and J. L. Bruyelle, "Fall on the track detection in public transport by artificial vision," Computers in Railways VI, 1998.

[10] M. Sorell and C. Krieg, M.L.and Stewart, "Fon-platform radar and optical track fusion," Conference Record of The Twenty-Seventh Asilomar Conference on, vol. 1, p. 268, 1993.

[11] M. I. Skolnik, Introduction to radar systems. McGraw-Hill, New York,, 1962.

[12] R. Johnson and H. Jasik, Antenna Engineering Handbook. McGrawHill, 1976.

[13] W. Ren, B.-Q. Gao, Z.-H. Xue, W.-M. Li, and B. Liu, "Full-wave analysis of broad wall slot's characteristics in rectangular waveguides," IEEE Trans. Antennas Propag., vol. 52, no. 9, pp. 2436 - 2444, sep. 2004.

[14] A. Knott, J. Shaeffer, and M. Tuley, Radar Cross Section: Its prediction, Measurement and reduction. Artech House, 1993.

[15] D. Andreuccetti, R. Fossi, and C. Petrucci, Calculation of the dielectric properties of human body tissues in the frequency range $10 \mathrm{~Hz}-100 \mathrm{GHz}$, http://niremf.ifac.cnr.it/tissprop/.

[16] C. E. Baum, "The singularity expansion method," in Transient Electromagnetic Fields, ser. Topics in Applied Physics, L. Felsen, Ed. Springer Berlin / Heidelberg, 1979, vol. 10, pp. 129-179, 10.1007/35400755348.

[17] N. Shuley and D. Longstaff, "Role of polarisation in automatic target recognition using resonance descriptions," Electronics Letters, vol. 40, no. 4, pp. $268-270$, feb. 2004.

[18] M. Van Blaricum and R. Mittra, "A technique for extracting the poles and residues of a system directly from its transient response," IEEE Trans. Antennas Propag., vol. 23, no. 6, pp. 777 - 781, nov. 1975. 